\begin{tabular}{|c|c|}
\hline \multirow{3}{*}{ 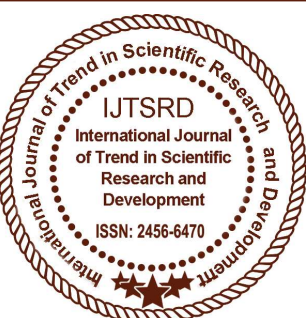 } & $\begin{array}{l}\text { International Journal of Trend in Scientific } \\
\text { Research and Development (IJTSRD) }\end{array}$ \\
\hline & International Open Access Journal \\
\hline & ISSN No: $2456-6470$ | www.ijtsrd.com | Volume - 2 | Issue - 3 \\
\hline
\end{tabular}

\title{
Three-Term Linear Fractional Nabla Difference Equation
}

G. Pushpalatha

Assistant Professor, Department of Mathematics, Vivekanandha College of Arts and Sciences for Women, Tiruchengode, Namakkal, Tamilnadu, India

\section{R. Ranjitha}

Research Scholar, Department of Mathematics, Vivekanandha College of Arts and Sciences for Women, Tiruchengode, Namakkal, Tamilnadu, India

\section{ABSTRACT}

In this present paper, a study on nabla difference equation and its third order linear fractional difference equation. A new generalized nabla difference equation is investigated from Three-term linear fractional nabla difference equation. A relevant example is proved and justify the proposed notions.

Keywords: Fractional difference operator, nabla difference equation, linear fractional, Third-term equation.

\section{Introduction}

In this present paper, we shall use the transform method to obtain solutions of a linear fractional nabla difference equation of the form

$t=1,2,3$..

$$
\nabla_{0}^{v} x(t)+C_{1} \nabla x(t)+C_{2} x(t)=g(t),
$$

Where $1<v \leq 2$. The fractional difference operator, $\nabla_{0}^{v}$ is of R-L type and the operator $\nabla_{0}^{\nu}$ is a RiemannLiouville fractional difference operator, is defined by,

If $\mu>0$, define the $\mu^{\text {th }}$-term of fractional sum by

$$
\nabla_{a}^{-\mu} x(t)=\sum_{s=a}^{t} \frac{(t-\rho(s))^{\overline{\mu-1}}}{\Gamma \mu} x(s)
$$

Where $\rho(s)=s-1$.

The aim for this paper is to develop and preserve the theory of linear fractional nabla difference equations as a corresponds of the theory of linear difference equations. We shall consider the three term equations, (1) is limited. An equation of the form

$$
\nabla_{0}^{2 \mu} x(t)+C_{1} \nabla_{0}^{\mu} x(t)+C_{2} x(t)=g(t)
$$

$t=1,2,3$..

is called a sequential fractional difference equation.

In general equation is,

$t=1,2,3$..,

$$
\nabla_{0}^{v_{2}} x(t)+C_{1} \nabla_{0}^{v_{1}} x(t)+C_{2} x(t)=g(t),
$$

Assume that $0<v_{1} \leq 1<v_{2} \leq 2$ as the only connection between $v_{1}$ and $v_{2}$. The operator of nabla is usually represents the backward difference operator and in this paper

$$
\begin{aligned}
& \nabla x(t)=x(t)-x(t-1), \\
& \nabla^{k} x(t)=\nabla \nabla^{k-1} x(t), \quad k=1,2,3 . .
\end{aligned}
$$

The raising factorial power function is defined below,

(6) $t^{\bar{\alpha}}=\frac{\Gamma(t+\alpha)}{\Gamma(\alpha)}$.

Then if $0 \leq m-1<v \leq m$, define by the RiemannLiouville fractional difference equation is

(7) $\nabla_{c}^{v} x(t)=\nabla^{m} \nabla_{c}^{v-m} x(t)$

Where $\nabla^{m}$ denotes the standard $m^{\text {th }}$ order nabla (backward) difference. 
In section 2, we shall use the transform method to (1) and we find out the solutions. And the same time we shall expressed as a sufficient condition as a function of $C_{1}$ and $C_{2}$ for convergent of the solutions.

In section 3, we apply the algorithm in the case of a solution is $2^{t}$ and verified independently that the series represents the known function.

For further studying in this previous area, we refer the reader to the article on two-term linear fractional nabla difference equation [7].

\section{Three-term Linear fractional nabla difference equation}

In this section, we describe an algorithm to form a solution of an initial value problem for a three-term linear fractional nabla difference equation of the form,

$$
\nabla_{0}^{v} x(t)+C_{1} \nabla x(t)+C_{2} x(t)=0
$$

$x(1)=c_{1} \quad$ for $\quad t=1,2,3 \ldots$

Where $1<v \leq 2$.

Apply the operator $N_{2}$ to the equation (8) we get, $N_{2}\left(\nabla_{0}^{v} x(t)+C_{1} \nabla x(t)+C_{2} x(t)\right)=0$

(9) $N_{2}\left(\nabla_{0}^{v} x(t)\right)+C_{1} N_{2}(\nabla x(t))+C_{2} N_{2} x(t)=0$

First, consider a term $N_{2}\left(\nabla_{0}^{v} x(t)\right)$ from (8) and use the result [7] we get,

$$
\text { "If } 1<v \leq 2 \text {, }
$$

$$
\begin{aligned}
N_{a+2}\left(\nabla_{a}^{v} f(t)\right)(s) & =s^{v} N_{a}(f(t))(s) \\
- & s(1-s)^{a-1} f(a)-(1-s)^{a} \nabla_{a}^{v-1} f(a+1) \\
= & s^{v} N_{a}(f(t))(s)-s(1-s)^{a-1} f(a) \\
& -(1-s)^{a}(f(a+1)-(v-1) f(a)) . "
\end{aligned}
$$$$
x(0)=c_{0},
$$$$
\text { ern }
$$

Next, consider the term $N_{2}(\nabla x(t))$ on (9) and also, we know that the result [7],

$$
\begin{gathered}
\text { "If } 0<v \leq 1, \quad N_{a+1}\left(\nabla_{a}^{v} f(t)\right)(s)=s^{v} N_{a}(f(t))(s) \\
-(1-s)^{a-1} f(a) . "
\end{gathered}
$$

Which implies

$$
\begin{aligned}
N_{2}(\nabla x(t)) & =N_{2}(\nabla x(t))+\nabla x(1)-\nabla x(1) \\
= & N_{1}(\nabla x(t))-\nabla x(1) \\
= & s N_{1}(x(t))-(1-s)^{-1} c_{0}-\left(c_{1}-c_{0}\right)
\end{aligned}
$$$$
=s N_{0}(x(t))-\frac{1}{(1-s)} c_{0}-c_{1}+c_{0} \frac{(1-s)}{(1-s)}
$$$$
=s N_{0}(x(t))-\frac{1}{(1-s)} c_{0}-c_{1}+\frac{1}{(1-s)} c_{0}-\frac{s}{(1-s)} c_{0}
$$

(11) $N_{2}(\nabla x(t))=s N_{0}(x(t))-\frac{s}{(1-s)} c_{0}-c_{1}$

Similarly, we consider the last term,

$$
N_{2}(x(t))=N_{2}(x(t))+c_{1}-c_{1}+\frac{1}{(1-s)} c_{0}-\frac{1}{(1-s)} c_{0}
$$$$
=N_{2}(x(t))+c_{1}+(1-s)^{-1} c_{0}-c_{1}+(1-s)^{-1} c_{0}
$$

\section{In particular}

(12) $N_{2}(x(t))=N_{0}(x(t))-c_{1}-(1-s)^{-1} c_{0}$

Substitute (10), (11) and (12) in (9) we get

$$
N_{2}\left(\nabla_{0}^{v} x(t)\right)+C_{1} N_{2}(\nabla x(t))+C_{2} N_{2} x(t)=0
$$

$s^{v} N_{0}(x(t))-s(1-s)^{-1} c_{0}-\left(c_{1}-(v-1) c_{0}\right)$

$+C_{1}\left(s N_{0}(x(t))-\frac{s}{(1-s)} c_{0}-c_{1}\right)$

$+C_{2}\left(N_{0}(x(t))-c_{1}-(1-s)^{-1} c_{0}\right)=0$

Which implies that,

$$
\begin{aligned}
N_{2}\left(\nabla_{0}^{v} x(t)\right)=s^{v} N_{0}(x(t))-s(1-s)^{0-1} x(0) & \left(s^{v}+C_{1} s+C_{2}\right) N_{0}(x(t))-\frac{1}{(1-s)}\left(s+C_{1} s+C_{2}\right) c_{0} \\
\left.-(1-s)^{0}(x(1))-(v-1) x(0)\right) & -\left(1+C_{1}+C_{2}\right) c_{1}-(1-v) c_{0}=0
\end{aligned}
$$

(10) $N_{2}\left(\nabla_{0}^{v} x(t)\right)=s^{v} N_{0}(x(t))$

$$
-s(1-s)^{-1} c_{0}-\left(c_{1}-(v-1) c_{0}\right)
$$


International Journal of Trend in Scientific Research and Development (IJTSRD) ISSN: 2456-6470

$$
\begin{aligned}
& \text { (13) } N_{0}(x(t))=\frac{\left(s+C_{1} s+C_{2}\right) c_{0}}{(1-s)\left(s^{v}+C_{1} s+C_{2}\right)} \\
& +\frac{\left(1+C_{1}+C_{2}\right) c_{1}+(1-v) c_{0}}{\left(s^{v}+C_{1} s+C_{2}\right)}
\end{aligned}
$$

Now take $\frac{1}{\left(s^{v}+C_{1} s+C_{2}\right)}=\frac{1}{s^{v}\left(1+\frac{C_{1} s}{s^{v}}+\frac{C_{2}}{s^{v}}\right)}$

$=\frac{1}{s^{v}\left(1+C_{1} s^{1-v}+\frac{C_{2}}{s^{v}}\right)}$

$$
\frac{1}{\left(s^{v}+C_{1} s+C_{2}\right)}=\frac{1}{s^{v}\left(1+C_{1} s^{1-\nu}\right)\left(1+\frac{C_{2}}{s^{v}\left(1+C_{1} s^{1-\nu}\right)}\right)} \text { In }
$$

general,

$$
\frac{1}{s^{v}+C_{1} s+C_{2}}=\sum_{n=0}^{\infty}(-1)^{n} s^{-v(n+1)} C_{2}^{n}\left(\frac{1}{1+C_{1} s^{1-v}}\right)^{n+1}
$$

Note that,

$$
\left(\frac{1}{1+C_{1} s^{1-v}}\right)^{n+1}=\sum_{m=n}^{\infty}(-1)^{m+n}\left(\frac{m}{n}\right)\left(C_{1} s^{1-v}\right)^{m-n}
$$

$$
\frac{1}{s^{v}+C_{1} s+C_{2}}=\sum_{m=0}^{\infty} \sum_{n=0}^{m}(-1)^{m}\left(\begin{array}{l}
m \\
n
\end{array}\right) C_{1}^{m-n} C_{2}^{n} s^{((1-v) m)-n-\nu}
$$

Since $s^{((1-v) m)-n-v}=N_{1}\left(\frac{t^{\overline{(v-1) m+n+(v-1)}}}{\Gamma((v-1) m+n+v)}\right)$

$$
=N_{0}\left(\frac{t^{\overline{(v-1) m+n+(v-1)}}}{\Gamma((v-1) m+n+v)}\right) \text {. }
$$

By using the result [7], “"

$$
A N_{1} f(t)=-\frac{A f(0)}{1-s}+A N_{0} f(t) \text { " }
$$

Now, the above equation is re-express $N_{1}$ as $N_{0}$, and we have,

$$
\frac{1}{s^{\nu}+C_{1} s+C_{2}}=\sum_{m=0}^{\infty} \sum_{n=0}^{m}(-1)^{m}\left(\begin{array}{l}
m \\
n
\end{array}\right) C_{1}^{m-n} C_{2}^{n}
$$

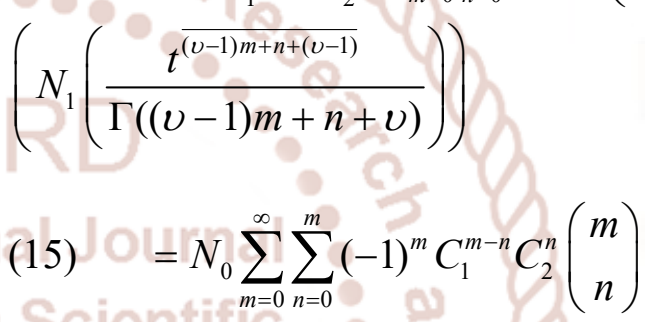

$$
\left(\frac{t^{\overline{(v-1) m+n+(v-1)}}}{\Gamma((v-1) m+n+v)}\right)
$$

It follows from the result [7]

" $N_{a} f(t+1)=(1-s)^{-1} N_{a+1} f(t)$ ".

We get

Which implies that, equation (15) we get,

$\frac{1}{s^{\nu}+C_{1} s+C_{2}}=\sum_{n=0}^{\infty} \sum_{m=n}^{\infty}(-1)^{m}\left(\begin{array}{l}m \\ n\end{array}\right) C_{1}^{m-n} s^{(1-v) m-n} C_{2}^{n} s^{-v(n+1)}$

$\frac{(1-s)^{-1}}{s^{v}+C_{1} s+C_{2}}=N_{0} \sum_{m=0}^{\infty} \sum_{n=0}^{m}(-1)^{m} C_{1}^{m-n} C_{2}^{n}\left(\begin{array}{l}m \\ n\end{array}\right)$

$$
\left(\frac{(t+1)^{\overline{(v-1) m+n+(v-1)}}}{\Gamma((v-1) m+n+v)}\right)
$$

$=\sum_{m=0}^{\infty} \sum_{n=0}^{m}(-1)^{m}\left(\begin{array}{l}m \\ n\end{array}\right) C_{1}^{m-n} C_{2}^{n} s^{m-m v-n+n v-n v-v}$

$$
\begin{aligned}
& \frac{1}{s^{\nu}+C_{1} s+C_{2}}=\sum_{m=0}^{\infty} \sum_{n=0}^{m}(-1)^{m}\left(\begin{array}{l}
m \\
n
\end{array}\right) C_{1}^{m-n} C_{2}^{n} s^{(1-v) m-n-v} \cdot \frac{s}{s} \\
& \frac{s}{s^{\nu}+C_{1} s+C_{2}}=\sum_{m=0}^{\infty} \sum_{n=0}^{m}(-1)^{m}\left(\begin{array}{l}
m \\
n
\end{array}\right) C_{1}^{m-n} C_{2}^{n} s^{(1-v) m-n-v+1} .
\end{aligned}
$$


International Journal of Trend in Scientific Research and Development (IJTSRD) ISSN: 2456-6470

Similarly, we get

$$
\begin{aligned}
& \frac{s}{s^{v}+C_{1} s+C_{2}}=\sum_{m=0}^{\infty} \sum_{n=0}^{m}(-1)^{m} C_{1}^{m-n} C_{2}^{n}\left(\begin{array}{c}
m \\
n
\end{array}\right) N_{0} \\
& \left(\frac{t^{\overline{(v-1) m+n+(v-2)}}}{\Gamma((v-1) m+n+(v-1))}\right) \\
& \text { and so } \\
& \frac{s(1-s)^{-1}}{s^{v}+C_{1} s+C_{2}}=N_{0} \sum_{m=0}^{\infty} \sum_{n=0}^{m}(-1)^{m} C_{1}^{m-n} C_{2}^{n}\left(\begin{array}{c}
m \\
n
\end{array}\right) \\
& \text { (17) } \quad\left(\frac{(t+1)^{\overline{(v-1) m+n+(v-2)}}}{\Gamma((v-1) m+n+(v-1))}\right)
\end{aligned}
$$

$+\left(1+C_{1}\right) c_{0} \sum_{m=0}^{\infty} \sum_{n=0}^{m}(-1)^{m} C_{1}^{m-n} C_{2}^{n}\left(\begin{array}{l}m \\ n\end{array}\right) \frac{(t+1)^{\overline{(v-1) m+n+(v-2)}}}{\Gamma((v-1) m+n+(v-1))}$

We consider an equation (13) we get

$$
\begin{aligned}
& N_{0} x(t)=\frac{\left(s+C_{1} s+C_{2}\right) c_{0}}{(1-s)\left(s^{v}+C_{1} s+C_{2}\right)} \\
& +\frac{\left(1+C_{1}+C_{2}\right) c_{1}+(1-v) c_{0}}{\left(s^{v}+C_{1} s+C_{2}\right)} \\
& \frac{(t+1)^{\overline{(v-1) m+n+(v-1)}}}{\Gamma((v-1) m+n+v)}=\frac{t^{\overline{(v-1) m+n+(v-1)}}}{\Gamma((v-1) m+n+v)} \\
& +\frac{(t+1)^{\overline{(v-1) m+n+(v-2)}}}{\Gamma((v-1) m+n+v-1)}
\end{aligned}
$$$$
+K \sum_{m=0}^{\infty} \sum_{n=0}^{m}(-1)^{m} C_{1}^{m-n} C_{2}^{n}\left(\begin{array}{l}
m \\
n
\end{array}\right) \frac{t^{\overline{(v-1) m+n+(v-1)}}}{\Gamma((v-1) m+n+v)}
$$

Where $K=\left(\left(1+C_{1}+C_{2}\right) c_{1}+(1-v) c_{0}\right)$.

To simplify this representation, note that

(18)

$$
\begin{array}{r}
N_{0} x(t)=\frac{C_{2} c_{0} \text { of }}{(1-s)\left(s^{v}+C_{1} s+C_{2}\right)} \\
+\frac{s\left(1+C_{1}\right) c_{0}}{(1-s)\left(s^{v}+C_{1} s+C_{2}\right)} \\
+\frac{\left(1+C_{1}+C_{2}\right) c_{1}+(1-v) c_{0}}{\left(s^{v}+C_{1} s+C_{2}\right)}
\end{array}
$$

Since

$$
\begin{aligned}
& \frac{(t+1)^{(v-1) m+n+(v-1)}}{\Gamma((v-1) m+n+v)}=\frac{\Gamma(t+1+(v-1) m+n+(v-1))}{\Gamma(t+1) \Gamma((v-1) m+n+v)} \\
& 0 n=t \frac{\Gamma(t+(v-1) m+n+(v-1))}{\Gamma(t+1) \Gamma((v-1) m+n+v)} \\
& 6=6470 \\
& +((v-1) m+n+(v-1)) \frac{\Gamma(t+(v-1) m+n+(v-1))}{\Gamma(t+1) \Gamma((v-1) m+n+v)}
\end{aligned}
$$

Thus, (19) can be expressed as

Substitute (15), (16) and (17) directly into (18) we get

$$
\begin{aligned}
& N_{0} x(t)=C_{2} c_{0} N_{0} \sum_{m=0}^{\infty} \sum_{n=0}^{m}(-1)^{m} C_{1}^{m-n} C_{2}^{n}\left(\begin{array}{l}
m \\
n
\end{array}\right) \frac{(t+1)^{(v-1) m+n+(v-1)}}{\Gamma((v-1) m+n+v)} \quad+K_{2} \sum_{m=0}^{\infty} \sum_{n=0}^{m}(-1)^{m} C_{1}^{m-n} C_{2}^{n}\left(\begin{array}{l}
m \\
n
\end{array}\right) \frac{t^{\overline{(v-1) m+n+(v-1)}}}{\Gamma((v-1) m+n+v)} \\
& +\left(1+C_{1}\right) c_{0} N_{0} \sum_{m=0}^{\infty} \sum_{n=0}^{m}(-1)^{m} C_{1}^{m-n} C_{2}^{n}\left(\begin{array}{l}
m \\
n
\end{array}\right) \frac{(t+1)^{(v-1) m+n+(v-2)}}{\Gamma((v-1) m+n+(v-1))}
\end{aligned}
$$$$
x(t)=K_{1} \sum_{m=0}^{\infty} \sum_{n=0}^{m}(-1)^{m} C_{1}^{m-n} C_{2}^{n}\left(\begin{array}{l}
m \\
n
\end{array}\right) \frac{(t+1)^{\overline{(v-1) m+n+(v-2)}}}{\Gamma((v-1) m+n+(v-1))}
$$

Where

$$
K_{1}=c_{0}\left(1+C_{1}+C_{2}\right),
$$

$K_{2}=K+c_{0} C_{2}=\left(1+C_{1}+C_{2}\right) c_{1}+\left(1-v+C_{2}\right) c_{0}$ Note

$+K N_{0} \sum_{m=0}^{\infty} \sum_{n=0}^{m}(-1)^{m} C_{1}^{m-n} C_{2}^{n}\left(\begin{array}{l}m \\ n\end{array}\right) \frac{t^{\overline{(v-1) m+n+(v-1)}}}{\Gamma((v-1) m+n+v)}$ that

$$
\sum_{\substack{m=0 \\
\text { and }}}^{\infty} \sum_{n=0}^{m}(-1)^{m} C_{1}^{m-n} C_{2}^{n}\left(\begin{array}{l}
m \\
n
\end{array}\right) \frac{(t+1)^{\overline{(v-1) m+n+(v-2)}}}{\Gamma((v-1) m+n+(v-1))}(22)
$$


$\sum_{m=0}^{\infty} \sum_{n=0}^{m}(-1)^{m} C_{1}^{m-n} C_{2}^{n}\left(\begin{array}{l}m \\ n\end{array}\right) \frac{t^{\overline{(v-1) m+n+(v-1)}}}{\Gamma((v-1) m+n+v)}$

(23)

are two linear independent solutions of (8). We give the details to obtain conditions for absolute convergence in (23) for fixed $t$.

First note that for each $t \geq 1, \frac{t^{\overline{(v-1) m+n+(v-1)}}}{\Gamma((v-1) m+n+v)}$ is an increasing function in $n$. Now consider the ratio $\frac{t^{\overline{(v-1) m+n+1+(v-1)}}}{\Gamma((v-1) m+n+1+v)} / \frac{t^{\overline{(v-1) m+n+(v-1)}}}{\Gamma((v-1) m+n+v)}$

Then

$$
\begin{aligned}
& \begin{array}{r}
\Gamma(t+(v-1) m+n+1+(v-1)) \\
\Gamma(t) \Gamma((v-1) m+n+1+v) \\
\Gamma(t) \Gamma((v-1) m+n
\end{array} \\
& \Gamma(t+(v-1) m+n \\
& =\frac{t+(v-1) m+n+(v-1)}{(v-1) m+n+v} \geq 1 \\
& \text { and the inequality is strict if } t>1 . \\
& \text { Thus, compare (23) to } \\
& \sum_{m=0}^{\infty} \frac{t^{(v m+(v-1))}}{\Gamma(v m+v)}\left|\sum_{n=0}^{m} C_{1}^{m-n} C_{2}^{n}\left(\begin{array}{l}
m \\
n
\end{array}\right)\right| \\
& =\sum_{m=0}^{\infty} \frac{(t)^{(v m+(v-1))}}{\Gamma(v m+v)}\left|C_{1}+C_{2}\right|^{m}
\end{aligned}
$$

and apply the ratio test. Thus, each of (22) and (23) are absolutely convergent if $\left|C_{1}+C_{2}\right|<1$.

\section{Description of known functions:}

We represent this method with an initial value problem for a classical second order finite difference equation. The unique solution of the initial value problem is $x(t)=2^{t}$, thus we obtain a series representation of $2^{t}$ as a linear combination of the forms (22) and (23).

Consider the initial value problem

(24) $\quad 10 \nabla^{2} x(t)-\nabla x(t)-2 x(t)=0, \quad t=2,3, .$. $x(0)=1, x(1)=2$.
Then, apply (13) with

$$
\begin{aligned}
& C_{1}=\frac{-1}{10}, \quad C_{2}=\frac{-1}{5}, \quad c_{0}=1, \quad c_{1}=2, \\
& v=2,
\end{aligned}
$$

To obtain

$$
\begin{aligned}
& N_{0} x(t)=\frac{(s+(-1 / 10) s+(-1 / 5)) 1}{(1-s)\left(s^{2}+(-1 / 10) s+(-1 / 5)\right)} \\
& +\frac{(1+(-1 / 10)+(-1 / 5)) 2+(1-2) 1}{\left(s^{2}+(-1 / 10) s+(-1 / 5)\right)}
\end{aligned}
$$$$
N_{0} x(t)=\frac{s-\frac{1}{10} s-\frac{1}{5}}{(1-s)\left(s^{2}-\frac{1}{10} s-\frac{1}{5}\right)}+\frac{2-\frac{2}{10}-\frac{2}{5}-1}{\left(s^{2}-\frac{1}{10} s-\frac{1}{5}\right)}
$$$$
N_{0} x(t)=\frac{\frac{9}{10} s-\frac{1}{5}}{(1-s)\left(s^{2}-\frac{1}{10} s-\frac{1}{5}\right)}+\frac{\frac{4}{10}}{\left(s^{2}-\frac{1}{10} s-\frac{1}{5}\right)}
$$

From (21), $K_{1}=1\left(1-\frac{1}{10}-\frac{1}{5}\right)=\frac{7}{10}$

$K_{2}=\left(1-\frac{1}{10}-\frac{1}{5}\right) 2+\left(1-2-\frac{1}{5}\right) 1=\left(\frac{7}{10}\right) 2-\frac{6}{5}$

$$
=\frac{1}{5} \text {. }
$$

and

$$
\begin{aligned}
& x(t)=\frac{7}{10} \sum_{m=0}^{\infty} \sum_{n=0}^{m}\left(\frac{1}{5}\right)^{n}\left(\frac{1}{10}\right)^{m-n}\left(\frac{m}{n}\right) \frac{(t+1)^{\overline{m+n}}}{\Gamma(m+n+1)} \\
& +\frac{1}{5} \sum_{m=0}^{\infty} \sum_{n=0}^{m}\left(\frac{1}{5}\right)^{n}\left(\frac{1}{10}\right)^{m-n}\left(\begin{array}{l}
m \\
n
\end{array}\right) \frac{(t)^{\overline{m+n}}}{\Gamma(m+n+2)} .
\end{aligned}
$$

\section{(25)}

The unique solution of (24) is $2^{t}$ and the series given in (25) absolutely convergent for all $t=0,1,2, .$. Write (25) as $x(t)=\frac{7}{10} C(t)+\frac{1}{5} D(t)$.

Where $C(t)=\sum_{m=0}^{\infty} \sum_{n=0}^{m}\left(\frac{1}{5}\right)^{n}\left(\frac{1}{10}\right)^{m-n}\left(\begin{array}{l}m \\ n\end{array}\right) \frac{(t+1)^{\overline{m+n}}}{\Gamma(m+n+1)}$

$$
D(t)=\sum_{m=0}^{\infty} \sum_{n=0}^{m}\left(\frac{1}{5}\right)^{n}\left(\frac{1}{10}\right)^{m-n}\left(\begin{array}{l}
m \\
n
\end{array}\right) \frac{(t)^{\overline{m+n}}}{\Gamma(m+n+2)} \mathrm{To}
$$

prove $x(t+1)=2 x(t)$, or 
International Journal of Trend in Scientific Research and Development (IJTSRD) ISSN: 2456-6470

$\frac{7}{10} C(t+1)+\frac{1}{5} D(t+1)=2\left(\frac{7}{10} C(t)+\frac{1}{5} D(t)\right)$

It is sufficient to show that

$D(t+1)=C(t)+D(t)$ and

$\frac{7}{10} C(t+1)=\left(\frac{6}{5} C(t)+\frac{1}{5} D(t)\right)$.

Now consider $D(t+1)$,

$D(t+1)=\sum_{m=0}^{\infty} \sum_{n=0}^{m}\left(\frac{1}{5}\right)^{n}\left(\frac{1}{10}\right)^{m-n}\left(\begin{array}{l}m \\ n\end{array}\right) \frac{(t+1)^{\overline{m+n+1}}}{\Gamma(m+n+2)}$

$=\sum_{m=0}^{\infty} \sum_{n=0}^{m}\left(\frac{1}{5}\right)^{n}\left(\frac{1}{10}\right)^{m-n}\left(\begin{array}{l}m \\ n\end{array}\right) \frac{\Gamma(t+1+m+n+1)}{\Gamma(t+1) \Gamma(m+n+2)}$

$=\sum_{m=0}^{\infty} \sum_{n=0}^{m}\left(\frac{1}{5}\right)^{n}\left(\frac{1}{10}\right)^{m-n}\left(\begin{array}{l}m \\ n\end{array}\right)(t+m+n+1)$

$$
\frac{\Gamma(t+m+n+1)}{\Gamma(t+1) \Gamma(m+n+2)}
$$

$=\sum_{m=0}^{\infty} \sum_{n=0}^{m}\left(\frac{1}{5}\right)^{n}\left(\frac{1}{10}\right)^{m-n}\left(\begin{array}{l}m \\ n\end{array}\right) t \frac{\Gamma(t+m+n+1)}{\Gamma(t+1) \Gamma(m+n+2)}$

$+\sum_{m=0}^{\infty} \sum_{n=0}^{m}\left(\frac{1}{5}\right)^{n}\left(\frac{1}{10}\right)^{m-n}\left(\begin{array}{l}m \\ n\end{array}\right)(m+n+1)$

$\Gamma(t+m+n+1)$

$\overline{\Gamma(t+1) \Gamma(m+n+2)}$

$$
D(t+1)=D(t)+C(t) \text {. }
$$

We have yet to obtain a direct approach to take $C(t+1)$. We begin by showing directly that $D(t)$ satisfies

$10 \nabla^{2} x(t)-\nabla x(t)-2 x(t)=0, \quad t=2,3,$.

Apply the power rule and

$D(t)=\sum_{m=0}^{\infty} \sum_{n=0}^{m}\left(\frac{1}{5}\right)^{n}\left(\frac{1}{10}\right)^{m-n}\left(\begin{array}{l}m \\ n\end{array}\right) \frac{t^{\overline{m+n+1}}}{\Gamma(m+n+2)}$

$\nabla D(t)=\sum_{m=0}^{\infty} \sum_{n=0}^{m}\left(\frac{1}{5}\right)^{n}\left(\frac{1}{10}\right)^{m-n}\left(\begin{array}{l}m \\ n\end{array}\right) \frac{t^{\overline{m+n}}}{\Gamma(m+n+1)}$

$$
\nabla^{2} D(t)=\sum_{m=1}^{\infty} \sum_{n=0}^{m}\left(\frac{1}{5}\right)^{n}\left(\frac{1}{10}\right)^{m-n}\left(\begin{array}{l}
m \\
n
\end{array}\right) \frac{t^{\overline{m+n-1}}}{\Gamma(m+n)}
$$

Thus,

$$
\begin{aligned}
& \nabla^{2} D(t)=\sum_{m=0}^{\infty} \sum_{n=0}^{m+1}\left(\frac{1}{5}\right)^{n}\left(\frac{1}{10}\right)^{m+1-n}\left(\begin{array}{c}
m+1 \\
n
\end{array}\right) \frac{t^{\overline{m+n}}}{\Gamma(m+n+1)} \\
& =\sum_{m=0}^{\infty}\left(\frac{1}{10} \sum_{n=0}^{m}\left(\frac{1}{5}\right)^{n}\left(\frac{1}{10}\right)^{m-n}\left(\left(\begin{array}{c}
m \\
n
\end{array}\right)+\left(\begin{array}{c}
m \\
n-1
\end{array}\right)\right)\right.
\end{aligned}
$$

$\left.\frac{t^{\overline{m+n}}}{\Gamma(m+n+1)}+\left(\frac{1}{5}\right)^{m+1} \frac{t^{\overline{2 m+1}}}{\Gamma(2 m+2)}\right)$

$=\sum_{m=0}^{\infty} \frac{1}{10} \sum_{n=0}^{m}\left(\frac{1}{5}\right)^{n}\left(\frac{1}{10}\right)^{m-n}\left(\begin{array}{l}m \\ n\end{array}\right) \frac{t^{\overline{m+n}}}{\Gamma(m+n+1)}$

$+\sum_{m=0}^{\infty} \sum_{n=0}^{m+1}\left(\frac{1}{5}\right)^{n}\left(\frac{1}{10}\right)^{m-n}\left(\begin{array}{c}m \\ n-1\end{array}\right) \frac{t^{\overline{m+n}}}{\Gamma(m+n+1)}$

$=\frac{1}{10} \nabla D(t)+\frac{1}{5} \sum_{m=0}^{\infty} \sum_{n=0}^{m}\left(\frac{1}{5}\right)^{n}\left(\frac{1}{10}\right)^{m-n}\left(\begin{array}{l}m \\ n\end{array}\right) \frac{t^{\overline{m+n+1}}}{\Gamma(m+n+2)}$

$\nabla^{2} D(t)=\frac{1}{10} \nabla D(t)+\frac{1}{5} D(t)$

A similar calculation shows that $C(t)$ satisfies

$10 \nabla^{2} x(t)-\nabla x(t)-2 x(t)=0, \quad t=2,3$.

We close by arguing that

$\frac{7}{10} C(t+1)=\left(\frac{6}{5} C(t)+\frac{1}{5} D(t)\right)$.

Simplify

$10 \nabla^{2} C(t+1)-\nabla C(t+1)-2 C(t+1)=0$

$10(C(t+1)-2 C(t)+C(t-1))$ 
Employ $C(t)=D(t+1)-D(t)$ and

$C(t-1)=D(t)-D(t-1)$ to obtain

$$
\begin{aligned}
& \left(\frac{7}{10} C(t)-C(t-1)\right)=\frac{7}{10}(D(t+1) \\
& -D(t))-(D(t)-D(t-1)) \\
& =\frac{7}{10}\left(D(t+1)-\frac{17}{10} D(t)+D(t-1)\right.
\end{aligned}
$$$$
=\left(\frac{7}{10}\left(D(t+1)-\frac{19}{10} D(t)+D(t-1)\right)+\frac{2}{10} D(t)\right.
$$

$\left(\frac{7}{10} C(t)-C(t-1)\right)=\frac{1}{5} D(t)$.

\section{References}

1. Agarwal, R.P., Difference Equations and Inequalities, Marcel Dekker, New York,1992.

2. Jagan Mohan Jonnalagadda, Asymptotic Behaviour of Linear Fractional Nabla Difference Equations, Inter. Jour. of Differ. Equ., (2017) 255265.

3. Jagan Mohan, J., Variation of Parameters for Nabla Fractional Difference Equations, Novi. Sad J. Math., (2014) 149-159.

4. Jagan Mohan Jonnalagadda., Numerical Solutions of Linear Fractional Nabla Difference Equations, Math. CA, (2016).

5. Jagan Mohan, J., Deekshitulu, G.V.S.R., Solutions of Nabla Fractional Difference Equations Using N-Transforms, Commun. Math. Stat. (2014) 1-16.

6. Jagan Mohan Jonnalagadda, Analysis of Nonlinear Fractional Nabla Difference Equations, Inter. Jour. of Analy. and App., (2015) 79-95.

7. Paul Eloe, $\mathrm{Zi}$ Ouyang., Multi-term Linear Fractional Nabla Difference Equations with Constant Coefficients, Inter. Jour. of Differ. Eqn, (2015) 91-106. 